\title{
La violencia de género en México, ¿en qué vamos?
}

\author{
Anel Cecilia Sánchez de los Monteros Arriaga
}

\begin{abstract}
Resumen
La violencia ejercida contra las mujeres debido a su desvalorización social y cultural es un tema preocupante en nuestro país y a nivel internacional. Se han tomado medidas para protegerlas y cambiar el panorama social, y si bien se ha avanzado en 2012 al tipificar el feminidicio como un delito, ${ }^{1}$ los esfuerzos deben ir más allá de las acciones de los gobiernos y los procesos judiciales que las regulan. Es necesario hacer un cambio cultural de fondo, dejar atrás la ideología patriarcal de nuestra sociedad, especialmente la latina, debido a que la mayoría de los países con índices más altos de feminicidios se encuentran precisamente en América Latina. ${ }^{2}$ La ideología heredada de una superioridad masculina es la base para que se cometan actos violentos de género en contra de las mujeres. Si esta ideología es cuestionada y, sobretodo, modificada, cada hombre y mujer podrá hacer valer sus derechos y libertades para decidir sobre sí misma, además de rodear a las mujeres de una sociedad que las proteja y respete.
\end{abstract}

Palabras clave: discriminación, feminicidio, machismo, patriarcado, violencia de género.

\section{GendeR Violence in MeXico, Where are We?}

\begin{abstract}
Violence against women due to their social and cultural devaluation is a matter of concern in our country and internationally. Measures have been taken in order to protect them and to change the social context, and although progress has been made in 2012 by criminalizing feminidice as a crime, efforts must go beyond the actions of governments and the judicial processes that regulate them. It is necessary to make a substantial change in culture, as to leave behind the patriarchal ideology of our society, especially the Latino one, because most of the countries with the highest rates of feminicides are found precisely in Latin America. The inherited ideology of male superiority is the basis for violent gender-based acts against women. If this ideology is questioned and, above all, modified, each man and woman will be able to assert their rights and freedom to decide on themselves, in addition to build a society in which women are protected and respected.
\end{abstract}

Nacional de

los Derechos Humanos (CNDH), 2014.

2 García, 2018.

Keywords: discrimination, feminicide, sexism, machismo, patriarchy, gender violence.

Dol: http://doi.org/10.22201/cuaieed.16076079e.2020.21.4.1 


\section{Anel Cecilia Sánchez de los Monteros Arriaga}

chila1997@hotmail.com https://orcid.org/0000-0002-7416-1267

Cursó la Licenciatura en Filosofía en la unam. Es miembro de la Red Mexicana de Mujeres filósofas. Se desempeña como Facilitadora parental certificada por Niños de Ahora; actualmente acompaña a padres y madres a descubrir su sabiduría interna para criar a sus hijos armoniosamente. Ha colaborado con distintas editoriales en sus áreas de ventas y mercadotecnia. Ahora ejerce como colaboradora freelance en el área Editorial como Correctora de Estilo y Redacción. 
"La violencia de género en México, ¿en qué vamos?"

\section{Introducción}

La violencia de género es aquella que afecta de una u otra manera a las mujeres, por el mero hecho de ser mujeres. Es atentar contra su integridad, libertad y dignidad. Algunos tipos de la violencia que se ejercen en contra de ellas son física, sexual y/o psicológica.

La violencia de género en México ha existido desde tiempos inmemoriales y responde a la cultura conocida como machista, con la que comulga gran parte de la sociedad. En 1993, la Asamblea General de las Naciones Unidas definió la violencia contra las mujeres como "todo acto de violencia basada en la pertenencia al sexo femenino, que tenga o pueda tener como resultado un daño o sufrimiento físico, sexual o psicológico para la mujer, así como las amenazas de tales actos, la coerción o la privación arbitraria de la libertad, tanto si se produce en la vida pública como en la vida privada" (Orden Jurídico Nacional, 1993).

En la década de los noventa, en México, se comenzaron a recabar datos e información sobre la violencia a las mujeres. A finales de 2019, el INEGI reportó un porcentaje de $66.1 \%$ de mujeres mayores de 15 años (30.7 millones) que fueron violentadas de alguna manera (siendo el más alto índice, de 43.9\%, ejercida por su esposo o pareja actual). En 2018 se registraron 3,752 defunciones por homicidio de mujeres, el más alto registrado en los últimos 29 años (1990-2018), lo que en promedio significa que fallecieron 10 mujeres diariamente por agresiones intencionales (INEGI, 2019). Diez años atrás, en 2009, tan sólo en el territorio de lo que entonces era el Distrito Federal, se registraron 128 feminicidios, que representan una tasa (por cada 100,000 mujeres) del 2.23. Y diez años más atrás, en 1999, 127 feminicidios, con una tasa del 2.85 (onu Mujeres, INMujeres México y Lx Legislatura Cámara de Diputados, 2011).

Desafortunadamente, la igualdad o equidad de géneroyla no discriminación no forman parte de la cultura en México. En los últimos años se han realizado muchos esfuerzos por parte de organizaciones internacionales, del gobierno local y de la sociedad civil para cambiar esta postura y desarrollar un concepto de equidad aplicable a nuestro contexto, pero actualmente aún seguimos en proceso de adoptarlo y adaptarlo a nuestra sociedad.

\section{Desarrollo}

En los estratos sociales, en general, se considera a las mujeres como seres humanos inferiores a los hombres (Quiñones, 2018). Engels opinaba que la diferencia entre una cortesana que alquila su cuerpo y una mujer casada era que esta última adquiere un "contrato" como esclava a cambio de un estatus social y estabilidad financiera (Cisterna, 2016). En la actualidad, esta inferioridad es notoria en la diferencia de sueldos entre hombres y mujeres que desempeñan una misma labor, que ocupan el mismo puesto. Especialmente en México, esta 
brecha salarial de género es muy marcada (Organización Internacional del Trabajo, 2019).

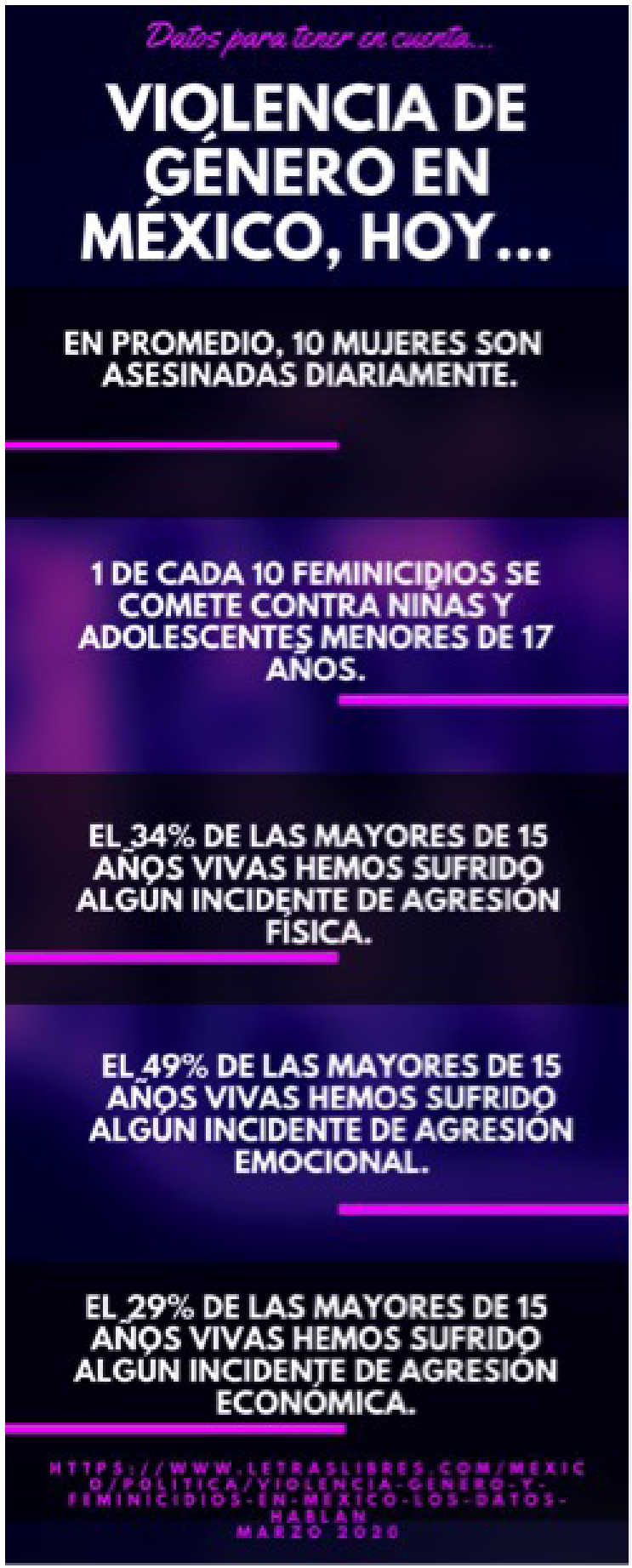

La inferioridad femenina es una idea tan arraigada en nuestra cultura y sociedad que se requerirán muchos años de educación familiar y académica para modificar esa idea equivocada sobre las mujeres, a una postura más justa para todos (Vázquez Pérez, 2008). Un sondeo realizado en 2017 por IPsos arrojó un resultado en el que 1 de cada 5 personas en el mundo (en países considerados del primer mundo), es decir aproximadamente $20 \%$ de la población mundial, considera que la mujer es inferior al hombre e incapaz de realizar ciertas actividades que el hombre sí puede.

La violencia de género afecta a toda la sociedad, especialmente a la latina, ya que se trata de países más tradicionalistas y donde las leyes difícilmente protegen a las mujeres. La sociedad que las rodea, por lo general, no cree en ellas. Y si nuestras mujeres son violentadas, nuestra sociedad también lo es, nuestra cultura y país. El índice de sexismo y violencia contra las mujeres se incrementa en grupos, zonas o países con menor nivel educativo, que se encuentran en guerra, con prácticas de riesgo (grupos delictivos), en adolescentes y en un nivel cultural bajo (IPSOS, 2017).

Se ha dado un gran paso al reconocer que la violencia de género existe con la tipificación del feminicidio como delito. Con la publicidad para combatirlo, se le ve como real y ha salido del ámbito privado, o sea, de competer únicamente a la familia y no "pasar de la puerta", a pasar a ser público. Así, se ha convertido en un tema que debe regularse legalmente y que figura en las principales preocupaciones a nivel mundial. Es verdad que la violencia de género sigue presente en la sociedad mexicana (ver infografía), en los anuncios piublicitarios donde se cosifica a la mujer, en los encabezados de desapariciones y muertes de mujeres, en la diferencia de sueldos por género... Pero, ha cambiado la manera en que la percibimos y ahora se trata como un tema reprobable, cuando antes se consideraba como algo "normal". En la actualidad es un delito aparte, hay protestas públicas por abusos o muertes de mujeres o niñas, se despliegan marchas o campañas para fomentar la denuncia y fortalecer la seguridad de las mujeres por parte del gobierno y la sociedad. La discriminación hacia la mujer, es 
"La violencia de género en México, ¿en qué vamos?" Anel Cecilia Sánchez de los Monteros Arriaga

decir, la exclusión o restricción a sus derechos por el simple hecho de ser mujer, se ha vuelto notoria en redes sociales y otros medios de comunicación masiva con la información casi en tiempo real de secuestros o feminicidios. Lo que aún no se ha logrado es cambiar la mentalidad ni afianzar los valores que promuevan el respeto por igual hacia todos los miembros que conforman la sociedad.

Gracias a organizaciones internacionales, como la OMS o la UNESE_CEPE pertenecientes a la onu, la violencia de género comenzó a considerarse un problema de salud pública. Al tratarla de esta manera, se empezaron a crear programas de toma de conciencia, establecer los lineamientos para detectarla, atenderla y buscar soluciones para erradicarla, por lo que ahora existen leyes internacionales que protegen a las mujeres y se ha dimensionado lo grave del asunto. Por ejemplo, está la Plataforma de Acción de Beijing, que busca "garantizar que las mujeres tengan el mismo derecho que los hombres a ser jueces, abogados, funcionarios de otro tipo en los tribunales, así como funcionarios policiales y funcionarios penitenciarios, entre otras cosas" (onu Mujeres, 2011).

La violencia hacia las mujeres se da en todos los ámbitos: en su integridad física, mental, moral; sucede dentro de su propia familia, en su vida laboral, económica y política; se ejerce a través de la explotación sexual o la prostitución forzada. Según onu Mujeres hay tres distintos tipos de violencia ejercidos contra las mujeres: física, sexual y psicológica. Desafortunadamente, la violencia contra la mujer no sólo sigue sin resolverse, sino que continúan creciendo los números de feminicidios, trata de personas con fines de explotación sexual y/o laboral, discriminación de distintos tipos, etcétera.

A pesar de que la teoría, la investigación y las legislaciones se han desarrollado para erradicar la violencia contra las mujeres, a veces parecen imposibles de llevar a la práctica y son algo muy lejano a nuestra realidad. Ejemplo de ello son los casos de feminicidios que han tenido una denuncia previa de la víctima hacia el agresor, quien, posteriormente, asesina a la víctima. Entre esa denuncia y la muerte de la víctima, no hay ninguna acción por parte de las autoridades, sólo un papeleo y un antecedente legal de los hechos. Es decir, no hay lineamientos para evitar el feminicidio o se archiva la denuncia porque no se continúa con el proceso. Así fue el caso de Ingrid Escamilla, el indignante feminicidio cometido al norte de la CDMx, que consternó a la población mexicana a principios de febrero de 2020.

En México, en particular, hombres y mujeres permiten y fomentan la violencia hacia las mujeres, al seguir los patrones de la educación cultural que los ha rodeado y formado. Así también educan a sus hijos y adoptan un rol machista en su familia, con la ideología práctica de que las mujeres están para servir, para ocuparse de las labores de la casa, de los hijos... Ellas son restringidas de las oportunidades de estudiar, de superarse y de ejercer un trabajo fuera de casa. Sus voces, opiniones y esfuerzos por destacar son minimizados. 
"La violencia de género en México, ¿en qué vamos?" Anel Cecilia Sánchez de los Monteros Arriaga

Es debido a esta misma ideología que las mujeres no denuncian cuando son víctimas de violencia, además del miedo a las represalias que pueden tomar en contra de ellas, así como a la revictimización de la que pueden ser objeto al momento de realizar la denuncia. Si se trata de una situación constante, les resulta sumamente difícil salir de ella: creen que no volverá a ocurrir o que "se lo ganaron" con su comportamiento, justifican al agresor y permiten que siga sucediendo. La ideología patriarcal sigue siendo dominante en nuestra cultura y eso nos sitúa a hombres y mujeres en un papel muy bien definido y del que es muy difícil despojarse. Cada paso que se ha dado hacia una ideología equitativa de género -cada vez que una mujer denuncia, que ejerce su derecho a vivir sin violencia - implica un enorme esfuerzo de por medio; uno por dejar atrás esas ideas y actitudes patriarcales, aún con todo lo que eso implica socialmente, y de enfrentar las leyes que no son favorables para las mujeres.

Las leyes y la sociedad civil necesitan hacer mucho más para alcanzar la equidad de género, para que se lleve a la práctica diaria y para que sea lo habitual en nuestra sociedad mexicana. En este sentido, la cultura de la denuncia aún no se ha difundido ni insertado lo suficiente para que cada caso de violencia sea atendido y solucionado. Los procesos judiciales no están estructurados para atender eficazmente las denuncias: para evitar la revictimización de las denunciantes y ofrecerles un entorno seguro, así como una solución real y práctica para protegerlas. Si el personal que recibe a las denunciantes no está calificado para esa labor, las víctimas no se abren a hablar sobre el agravio que les hicieron. De hecho, en muchas ocasiones, además del daño que ya han recibido, este mismo personal les hace sentir culpa por lo sucedido, como si ellas fueran quienes provocaron o incitaron a su agresor a atacarlas.

Cabe resaltar que los actos de violencia que se cometen contra las mujeres, como el feminicidio, no han sido tipificados penalmente en todos los países o es algo que recién ha ocurrido en otros. Algunas leyes han logrado establecer penalmente los delitos cometidos contra las mujeres y sus correspondientes sanciones, pero la aplicación de las leyes es muy ineficiente en México, así que, en la práctica, dichos delitos siguen quedando impunes. Por un lado, los agresores saben que es altamente probable que no sean sancionados y, por tal motivo, siguen cometiendo las agresiones. Por el otro, las víctimas prefieren callar precisamente porque conocen que el sistema no aplica las sanciones correspondientes y, además, tienen temor de que la ley no las proteja y a sufrir represalias por haber denunciado. Se cree que un porcentaje muy alto, prácticamente el equivalente al número de denuncias, permanece sin denuncia alguna (Expansión Política, 2020).

Las mujeres y todos tienen derecho a una vida libre de violencia. Cuando esta última es ejercida contra ellas, sus afectaciones pueden traer consecuencias de todo tipo (emocionales, de salud física y mental, y laboral), incluso la muerte. La violencia contra las mujeres las afecta en un principio a ellas, e indirectamente 
"La violencia de género en México, ¿en qué vamos?" Anel Cecilia Sánchez de los Monteros Arriaga

Gráfica 1. Feminicidios cometidos en 2018, en gran parte de Latinoamérica y algunos países de Europa, así como la tasa de los mismos, por cada 100,000 mujeres. Elaboración propia con datos de CEPAL, 2019; La Vanguardia, 2019 y. AFP, 2020. a sus familias, a la comunidad a la que pertenecen y a la sociedad de su país. La violencia que viven llega a convertirse en un padecimiento generacional, porque sus hijos y familiares sufren con ellas al momento de enterarse del suceso. Asimismo, sucede en los casos en que los hijos son espectadores del acto violento, pues se ha observado que vivir estas experiencias, por ejemplo, de violencia intrafamiliar, aumenta el riesgo de, en el futuro, convertirse en víctimas o en agresores. Además, es más probable que las mujeres que han padecido algún tipo de maltrato sexual y/o físico sufran un aborto u otros problemas de salud reproductiva, depresión o pensamientos suicidas, con respecto a aquellas que no lo han experimentado.

Es alarmante el índice de violencia de género que existe en México. De fondo, no se ha logrado cambiar la imagen y el concepto que se tiene de las mujeres y de su valor en la sociedad. Se le concibe como un ser frágil, dependiente y que está a merced de los demás, de su familia, su pareja, sus hijos... Se encuentra tan desvalorizada que es atacada por personas tan cercanas como su propia familia o tan desconocidos como cualquiera que pasa delante de ella por la calle. Se cree que la mujer es una extensión del hombre, que si está "sola" no es valiosa, que obtiene el "título nobiliario" de llamarla "Señora" sólo si tiene un hombre en su vida "que la respalda". Se piensa que debe ser sumisa y callada, que su inteligencia es menor que la del varón, que está afectada por sus emociones y no es capaz de pensar con discernimiento; a veces se le ve como un objeto o un trofeo, carente de criterio, de opiniones y de sueños.

\section{Conclusiones}

La violencia de género es un problema que aqueja a todo el mundo, pero en Latinoamérica $y$, por ende, en México, representa un problema gravísimo y excesivamente preocupante. En estos países se concentran las tasas más altas de muerte por violencia de género. Los feminicidios se han convertido en parte del panorama habitual de las noticias, de los comentarios diarios y se encuentran a la vuelta de la esquina. No ocurren con mayor frecuencia en las comunidades más marginadas y con menos educación y recursos, como equivocadamente se cree, pasan en todos los niveles educativos, ideológicos y financieros.

Latinoamérica tiene el mayor número de casos de violencia sexual por parte de alguien que no es su pareja y un considerable número de casos de violencia ejercida por parejas. En algunos países latinos, incluyendo México, los 
"La violencia de género en México, ¿en qué vamos?" Anel Cecilia Sánchez de los Monteros Arriaga

feminicidos o actos violentos en contra de las mujeres tienen que ver, en gran parte, con el crimen organizado. En los países latinoamericanos la violencia de género sigue estando más presente que en otros de Europa como Italia, España o Alemania (ver gráfica 1).

La cultura patriarcal en la que a la mujer se le resta valor, poder y participación con respecto a los hombres disminuirá en la medida en que las mujeres sean empoderadas por su país, su sociedad y por ellas mismas. De esta manera se puede alcanzar una equidad de género real, que permita a todos vivir libres de violencia. Cambiar gran parte de la base de la cultura, la misma que tiene México y los demás países latinos, es la gran tarea que tienen las organizaciones gubernamentales, las asociaciones y la sociedad civil, si realmente se quiere alcanzar la equidad de género. Transformar hacia la equidad todo aquello que resta derechos a las mujeres debe ser el objetivo principal de esta lucha para protegerlas.

Implementar programas regionales y nacionales para la difusión de las ideas de igualdad es una de las principales tareas. La humanidad merece mujeres completas, cuidadas y protegidas. Conseguirlo es deber conjunto del gobierno, las asociaciones y la sociedad civil. Para seguir avanzando, ¿qué más podemos hacer por nuestras mujeres?

\section{Referencias}

- AFP Actual / AFP Argentina (2020, 11 de marzo) . ¿Cuántas víctimas de feminicidio hubo en América, Italia, España y Francia? https://factual.afp.com/cuantasvictimas-de-feminicidio-hubo-en-2019-en-america-italia-espana-y-francia

* Cisterna, Rodrigo. (2016, septiembre). Marx y Engels: Selección de Textos sobre la Mujer. https://www.marxists.org/espanol/m-e/selecciones/m e mujer.htm

* Comisión Nacional de los Derechos Humanos (CNDH). (2014, 24 de noviembre). Anexo Tipificación Feminicidio. https://www.cndh.org.mx/sites/all/doc/Programas/ mujer/6 MonitoreoLegislacion/6.9/A/tipificacionFeminicidioAnexo 2014nov05.pdf

* Expansión Política. (2020, 22 de enero). El 99.7\% de los delitos de violencia sexual contra mujeres no se denuncia. https://politica.expansion.mx/mexico/2020/01/22/ el-99-7-de-los-delitos-de-violencia-sexual-contra-mujeres-no-se-denuncia

* García, Ana Karen. (2018,20 de noviembre). 14 de los 25 países con más feminicidios se ubican en América Latina. El Economista. https://www.eleconomista.com. $\mathrm{mx} /$ politica/Violencia-de-genero-14-de-los-25-paises-del-mundo-con-masfeminicidios-se-ubican-en-America-Latina--20181120-0048.html

* INEGI. (2019, 21 de noviembre). Estadísticas a propósito del día internacional de la eliminación de la violencia contra la mujer (25 de noviembre) (Comunicado de prensa núm. 592/19). https://www.inegi.org.mx/contenidos/saladeprensa/ aproposito/2019/Violencia2019 Nal.pdf

- IPsos. (2017, 8 de marzo). Feminismo e igualdad de género en el mundo. https://www. ipsos.com/es-ve/feminismo-e-igualdad-de-genero-alrededor-del-mundo 
"La violencia de género en México, ¿en qué vamos?" Anel Cecilia Sánchez de los Monteros Arriaga

* La Vanguardia. Vida (2019, 25 de noviembre). La violencia de género dejó 122 mujeres muertas en Alemania en 2018. https://www.lavanguardia.com/ vida/20191125/471846035764/la-violencia-de-genero-dejo-122-mujeresmuertas-en-alemania-en-2018.html

* Observatorio de Igualdad de Género, CEPAL (Comisión Económica para América Latina y el Caribe) 2019, 02 de noviembre. Feminicidio https://oig.cepal.org/es/ indicadores/feminicidio

* Observatorio de Igualdad de Género, CEPAL (Comisión Económica para América Latina y el Caribe) 2019, 02 de noviembre. La medición del feminicidio o femicidio - Observatorio de Igualdad de Género. https://oig.cepal.org/sites/default/files/ femicidio web.pdf

* onv Mujeres. (2011, 30 de diciembre). Principales leyes, instrumentos y acuerdos internacionales y regionales. https://www.endvawnow.org/es/articles/1125principales-leyes-instrumentos-y-acuerdos-internacionales-y-regionales.html

* onu Mujeres, INMujeres México y Lxi Legislatura Cámara de Diputados. (2011, 8 de marzo). Feminicidio en México. Aproximación, tendencias y cambios, 19852009 [Cuadro 17, página 79]. http://cedoc.inmujeres.gob.mx/documentos download/00 feminicMx1985-2009.pdf

- Orden Jurídico Nacional. (1993, 20 de diciembre). Declaración sonbre la Eliminación de la Violencia contra la Mujer. Gobierno de México. http://www.ordenjuridico.gob. mx/TratInt/Derechos\%20Humanos/INST\%2018.pdf

* Organización Internacional del Trabajo. (2019). ¿Quétan grande es la brecha salarial de género en su país? (Informe Mundial sobre salarios 2018/2019). https://www.ilo.org/ global/about-the-ilo/multimedia/maps-and-charts/enhanced/WCMS 6508721 lang--es/index.htmQuiñones, Laura. (2018, 14 de febrero). Las mujeres están por debajo de los hombres en todos los indicadores de desarrollo sostenible. Noticias onu, Naciones Unidas. https://news.un.org/es/story/2018/02/1427081

* Vázquez Pérez, Teresa. (2008). Diagnóstico sobre la violencia de género y social en la Ciudad de México. InDESOL; Observatorio. Violencia de género y social en la Ciudad de México; CIPE. https://www.gob.mx/cms/uploads/attachment/file/159755/2010 OVSYG DF 3 CIPE.pdf

\section{Sitios de interés}

- Hechos y cifras: Acabar con la violencia contra mujeres y niñas. ONU Mujeres

* La violencia feminicida en México Aproximaciones ytendencias. SEGOB, INMujeres y ONU Mujeres

* Violencia de Género. Fondo de Población de las Naciones Unidas

- Latinoamérica es la region más peligrosa del mundo para las mujeres. ONU 


\section{Cómo citar el artículo}

* Sánchez de los Monteros Arriaga, Anel Cecilia. (2020). La violencia de género en México, ¿en qué vamos? Revista Digital Universitaria (RDU), 21(4) julio-agosto. Dol: http://doi.org/10.22201/cuaieed.16076079e.2020.21.4.1

Recepción: 15/05/2019. Aceptación: 21/02/2020. 